\title{
PROPERTIES OF HARMONIC FUNCTIONS WHICH ARE CONVEX OF ORDER $\beta$ WITH RESPECT TO SYMMETRIC POINTS
}

\author{
AINI JANTENG AND SUZEINI ABDUL HALIM
}

\begin{abstract}
Let $\mathscr{H}$ denote the class of functions $f$ which are harmonic and univalent in the open unit $\operatorname{disc} D=\{z:|z|<1\}$ This paper defines and investigates a family of complex-valued harmonic functions that are orientation preserving and univalent in $\mathscr{D}$ and are related to the functions convex of order $\beta(0 \leq \beta<1)$, with respect to symmetric points. We obtain coefficient conditions, growth result, extreme points, convolution and convex combinations for the above harmonic functions.
\end{abstract}

\section{Introduction}

A continuous complex-valued function $f=u+i v$ defined in a simply connected complex domain $E$ is said to be harmonic in $E$ if both $u$ and $v$ are real harmonic in $E$. There is a close inter-relation between analytic functions and harmonic functions. For example, for real harmonic functions $u$ and $v$ there exist analytic functions $U$ and $V$ so that $u=\operatorname{Re}(U)$ and $v=\operatorname{Im}(V)$. Then

$$
f(z)=h(z)+\overline{g(z)}
$$

where $h$ and $g$ are, respectively, the analytic functions $(U+V) / 2$ and $(U-V) / 2$. In this case, the Jacobian of $f=h+\bar{g}$ is given by

$$
J_{f}=\left|h^{\prime}(z)\right|^{2}-\left|g^{\prime}(z)\right|^{2} .
$$

The mapping $z \mapsto f(z)$ is orientation preserving and locally one-to-one in $E$ if and only if $J_{f}>0$ in $E$. The function $f=h+\bar{g}$ is said to be harmonic univalent in $E$ if the mapping $z \mapsto f(z)$ is orientation preserving, harmonic and one-to-one in $E$. We call $h$ the analytic part and $g$ the co-analytic part of $f=h+\bar{g}$.

Let $\mathscr{H}$ denote the class of functions $f=h+\bar{g}$ which are harmonic and univalent in $\mathscr{D}$ the unit disc with the normalization

$$
h(z)=z+\sum_{n=2}^{\infty} a_{n} z^{n}, \quad g(z)=\sum_{n=1}^{\infty} b_{n} z^{n},\left|b_{1}\right|<1 .
$$

Received January 23, 2008; revised May 6, 2008.

2000 Mathematics Subject Classification. Primary 30C45.

Key words and phrases. Harmonic functions, convex of order $\beta$ with respect to symmetric points, coefficient estimates. 
There have been rigorous works conducted on the class of complex harmonic functions $\mathscr{H}$. In [2], Sheil-Small and Clunie obtained properties for functions in this class. Since then, there have been other subclasses of $\mathscr{H}$ that were developed. These include the class of harmonic functions starlike in the unit disc $\mathscr{D}$ (see Jahangiri [6]) and convex harmonic functions in $\mathscr{D}$ (see Kim et al. [7]). Other related works also appear in [1], [5] and [10]. Silverman in [9] formed the class $\overline{\mathscr{H}}$, a subclass of $\mathscr{H}$ which consists harmonic functions with negative coefficients. The class $\overline{\mathscr{H}}$ is defined below.

Let $\overline{\mathscr{H}}$ be the subclass of $\mathscr{H}$ consisting of functions $f=h+\bar{g}$ so that the functions $h$ and $g$ take the form

$$
h(z)=z-\sum_{n=2}^{\infty}\left|a_{n}\right| z^{n}, \quad g(z)=-\sum_{n=1}^{\infty}\left|b_{n}\right| z^{n}, \quad\left|b_{1}\right|<1 .
$$

Another interesting class of functions is the class of functions starlike with respect to symmetric points, first introduced by Sakaguchi in [8], whereby it was proven that this class forms a subclass of the univalent class of functions. Das and Singh [4] further generalised this idea and introduced the class of functions which are convex with respect to symmetric points. The authors, by combining defined the new class of functions as follows :

Definition 1.1. Let $f \in \mathscr{H}$. Then $f \in \mathscr{H} \mathscr{C}_{s}(\beta)$ is said to be harmonic convex of order $\beta$, with respect to symmetric points, if and only if, for $0 \leq \beta<1$,

$$
\operatorname{Re}\left\{\frac{2\left[z^{2} h^{\prime \prime}(z)+z h^{\prime}(z)+\overline{z^{2} g^{\prime \prime}(z)+z g^{\prime}(z)}\right]}{z h^{\prime}(z)-\overline{z g^{\prime}(z)}+z h^{\prime}(-z)-\overline{z g^{\prime}(-z)}}\right\} \geq \beta .
$$

Also, we let $\overline{\mathscr{C}} \mathscr{C}_{s}(\beta)=\mathscr{H}_{\mathscr{C}_{s}}(\beta) \cap \overline{\mathscr{H}}$.

In this paper, the authors are motivated to determine properties of this new class which include coefficients results, growth bounds, extreme points and convolution properties.

The following theorem proved by Darus et al. in [3] will be used throughout in this paper.

Theorem 1.1.([3]) Let $f=h+\bar{g}$ with $h$ and $g$ of the form (1). If

$$
\sum_{n=2}^{\infty} \frac{\left(2 n-\beta\left(1-(-1)^{n}\right)\right)}{2(1-\beta)}\left|a_{n}\right|+\sum_{n=1}^{\infty} \frac{\left(2 n+\beta\left(1-(-1)^{n}\right)\right)}{2(1-\beta)}\left|b_{n}\right| \leq 1
$$

then $f$ is harmonic, orientation preserving, univalent in $\mathscr{D}$ and $f \in \mathscr{H}_{S}^{\star}(\beta)$.

Putting $f(-z)=-f(z)$ gives Jahangiri's result in [6]. 


\section{Results}

We begin the results with a sufficient coefficient condition for functions in $\mathscr{H}^{C} \mathscr{C}_{s}(\beta)$.

Theorem 2.1. Let $f=h+\bar{g}$ be of the form (1). If

$$
\sum_{n=2}^{\infty} \frac{n\left(2 n-\beta\left(1-(-1)^{n}\right)\right)}{2(1-\beta)}\left|a_{n}\right|+\sum_{n=1}^{\infty} \frac{n\left(2 n+\beta\left(1-(-1)^{n}\right)\right)}{2(1-\beta)}\left|b_{n}\right| \leq 1,
$$

then $f$ is harmonic, orientation preserving, univalent in $\mathscr{D}$ and $f \in \mathscr{H}^{C} \mathscr{C}_{s}(\beta)$.

Remark. By putting $f(-z)=-f(z)$, we will obtain the result given by Kim et al. in [7].

Proof. Since $\frac{\left(2 n-\beta\left(1-(-1)^{n}\right)\right)}{2(1-\beta)} \leq \frac{n\left(2 n-\beta\left(1-(-1)^{n}\right)\right)}{2(1-\beta)}$ and $\frac{\left(2 n+\beta\left(1-(-1)^{n}\right)\right)}{2(1-\beta)} \leq \frac{n\left(2 n+\beta\left(1-(-1)^{n}\right)\right)}{2(1-\beta)}$, it fol-

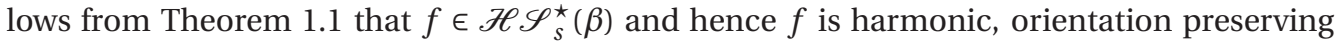
and univalent in $\mathscr{D}$. Now, we only need to show that if (5) holds then

$$
\operatorname{Re}\left\{\frac{2\left[z^{2} h^{\prime \prime}(z)+z h^{\prime}(z)+\overline{z^{2} g^{\prime \prime}(z)+z g^{\prime}(z)}\right]}{z h^{\prime}(z)-\overline{z g^{\prime}(z)}+z h^{\prime}(-z)-\overline{z g^{\prime}(-z)}}\right\}=\operatorname{Re} \frac{A(z)}{B(z)} \geq \beta .
$$

Using the fact that $\operatorname{Re}(w) \geq \beta$ if and only if $|1-\beta+w| \geq|1+\beta-w|$, it sufficies to show that

$$
|A(z)+(1-\beta) B(z)|-|A(z)-(1+\beta) B(z)| \geq 0,
$$

where

$$
A(z)=2\left[z^{2} h^{\prime \prime}(z)+z h^{\prime}(z)+\overline{z^{2} g^{\prime \prime}(z)+z g^{\prime}(z)}\right]
$$

and

$$
B(z)=z h^{\prime}(z)-\overline{z g^{\prime}(z)}+z h^{\prime}(-z)-\overline{z g^{\prime}(-z)} .
$$

Substituting for $A(z)$ and $B(z)$ in (6), we obtain

$$
\begin{aligned}
\mid A(z) & +(1-\beta) B(z)|-| A(z)-(1+\beta) B(z) \mid \\
= & \mid(2+2(1-\beta)) z+\sum_{n=2}^{\infty} n\left[2 n+(1-\beta)\left(1-(-1)^{n}\right)\right] a_{n} z^{n} \\
& +\sum_{n=1}^{\infty} n\left[2 n-(1-\beta)\left(1-(-1)^{n}\right)\right] b_{n} \bar{z}^{n}|-|(2-2(1+\beta)) z \\
& +\sum_{n=2}^{\infty} n\left[2 n-(1+\beta)\left(1-(-1)^{n}\right)\right] a_{n} z^{n}+\sum_{n=1}^{\infty} n\left[2 n+(1+\beta)\left(1-(-1)^{n}\right)\right] b_{n} \bar{z}^{n} \mid \\
\geq & (2+2(1-\beta))|z|-\sum_{n=2}^{\infty} n\left[2 n+(1-\beta)\left(1-(-1)^{n}\right)\right]\left|a_{n}\right||z|^{n} \\
& \quad-\sum_{n=1}^{\infty} n\left[2 n-(1-\beta)\left(1-(-1)^{n}\right)\right]\left|b_{n}\right||z|^{n}-(2(1+\beta)-2)|z| \\
& \quad-\sum_{n=2}^{\infty} n\left[2 n-(1+\beta)\left(1-(-1)^{n}\right)\right]\left|a_{n}\right||z|^{n}-\sum_{n=1}^{\infty} n\left[2 n+(1+\beta)\left(1-(-1)^{n}\right)\right]\left|b_{n}\right||z|^{n}
\end{aligned}
$$




$$
\begin{aligned}
& \geq 4(1-\beta)|z|\left\{1-\sum_{n=2}^{\infty} \frac{n\left[2 n-\beta\left(1-(-1)^{n}\right)\right]}{2(1-\beta)}\left|a_{n}\right|-\sum_{n=1}^{\infty} \frac{n\left[2 n+\beta\left(1-(-1)^{n}\right)\right]}{2(1-\beta)}\left|b_{n}\right|\right\} \\
& \geq 0, \quad b y(5) .
\end{aligned}
$$

The harmonic functions

$$
f(z)=z+\sum_{n=2}^{\infty} \frac{2(1-\beta)}{n\left[2 n-\beta\left(1-(-1)^{n}\right)\right]} x_{n} z^{n}+\sum_{n=1}^{\infty} \frac{2(1-\beta)}{n\left[2 n+\beta\left(1-(-1)^{n}\right)\right]} \bar{y}_{n} \bar{z}^{n},
$$

where $\sum_{n=2}^{\infty}\left|x_{n}\right|+\sum_{n=1}^{\infty}\left|y_{n}\right|=1$, show that the coefficient bound given in Theorem 2.1 is sharp.

The functions of the form (7) are in $\mathscr{P} \mathscr{C}_{s}(\beta)$ since

$$
\sum_{n=2}^{\infty} \frac{n\left(2 n-\beta\left(1-(-1)^{n}\right)\right)}{2(1-\beta)}\left|a_{n}\right|+\sum_{n=1}^{\infty} \frac{n\left(2 n+\beta\left(1-(-1)^{n}\right)\right)}{2(1-\beta)}\left|b_{n}\right|=\sum_{n=2}^{\infty}\left|x_{n}\right|+\sum_{n=1}^{\infty}\left|y_{n}\right|=1 .
$$

Next we show that the bound (5) is also necessary for functions in $\overline{\mathscr{C}^{C}}{ }_{s}(\beta)$.

Theorem 2.2. Let $f=h+\bar{g}$ with $h$ and $g$ of the form (2). Then $f \in \overline{\mathscr{P}} \mathscr{C}_{s}(\beta)$ if and only if

$$
\sum_{n=2}^{\infty} \frac{n\left(2 n-\beta\left(1-(-1)^{n}\right)\right)}{2(1-\beta)}\left|a_{n}\right|+\sum_{n=1}^{\infty} \frac{n\left(2 n+\beta\left(1-(-1)^{n}\right)\right)}{2(1-\beta)}\left|b_{n}\right| \leq 1
$$

Proof. In view of Theorem 2.1, we only need to show that $f$ is not in $\overline{\mathscr{C}} \mathscr{C}_{s}(\beta)$ if condition (8) does not hold. We note that a necessary and sufficient condition for $f=h+\bar{g}$ given by (2) to be in $\mathscr{H}^{C} \mathscr{C}_{s}(\beta)$ is that the coefficient condition (3) to be satisfied. Equivalently, we must have

$$
\begin{aligned}
& \operatorname{Re}\left\{\frac{2\left[z^{2} h^{\prime \prime}(z)+z h^{\prime}(z)+\overline{z^{2} g^{\prime \prime}(z)+z g^{\prime}(z)}\right]}{z h^{\prime}(z)-\overline{z g^{\prime}(z)}+z h^{\prime}(-z)-\overline{z g^{\prime}(-z)}}-\beta\right\} \\
& =\operatorname{Re}\left\{\frac{2 z-\sum_{n=2}^{\infty} 2 n^{2}\left|a_{n}\right| z^{n}-\sum_{n=1}^{\infty} 2 n^{2}\left|b_{n}\right| \bar{z}^{n}}{2 z-\sum_{n=2}^{\infty} n\left(1-(-1)^{n}\right)\left|a_{n}\right| z^{n}+\sum_{n=1}^{\infty} n\left(1-(-1)^{n}\right)\left|b_{n}\right| \bar{z}^{n}}-\beta\right\} \\
& =\operatorname{Re}\left\{\frac{2(1-\beta)-\sum_{n=2}^{\infty} n\left[2 n-\beta\left(1-(-1)^{n}\right)\right]\left|a_{n}\right| z^{n-1}-\frac{\bar{z}}{z} \sum_{n=1}^{\infty} n\left[2 n+\beta\left(1-(-1)^{n}\right)\right]\left|b_{n}\right| \bar{z}^{n-1}}{2-\sum_{n=2}^{\infty} n\left(1-(-1)^{n}\right)\left|a_{n}\right| z^{n-1}+\frac{\bar{z}}{z} \sum_{n=1}^{\infty} n\left(1-(-1)^{n}\right)\left|b_{n}\right| \bar{z}^{n-1}}\right\} \\
& \geq 0 .
\end{aligned}
$$

The above condition must hold for all values of $z,|z|=r<1$. Upon choosing the values of $z$ on the positive real axis where $0 \leq z=r<1$, the above inequality reduces to

$$
\frac{2(1-\beta)-\sum_{n=2}^{\infty} n\left[2 n-\beta\left(1-(-1)^{n}\right)\right]\left|a_{n}\right| r^{n-1}-\sum_{n=1}^{\infty} n\left[2 n+\beta\left(1-(-1)^{n}\right)\right]\left|b_{n}\right| r^{n-1}}{2-\sum_{n=2}^{\infty} n\left(1-(-1)^{n}\right)\left|a_{n}\right| r^{n-1}+\sum_{n=1}^{\infty} n\left(1-(-1)^{n}\right)\left|b_{n}\right| r^{n-1}} \geq 0 .
$$

If condition (8) does not hold then the numerator in (9) is negative for $r$ sufficiently close to 1 . Thus there exists $z_{0}=r_{0}$ in $(0,1)$ for which the quotient (9) is negative. This contradicts the required condition for $f \in \overline{\mathscr{H}} \mathscr{C}_{s}(\beta)$ and so the proof is complete. 
The growth result for functions in $\overline{\mathscr{H}} \mathscr{C}_{s}(\beta)$ is discussed in the following theorem.

Theorem 2.3. If $\in \in \overline{\mathscr{H}} \mathscr{C}$ s then

$$
|f(z)| \leq\left(1+\left|b_{1}\right|\right) r+\left(\frac{1-\beta}{4}-\frac{1+\beta}{4}\left|b_{1}\right|\right) r^{2}, \quad|z|=r<1
$$

and

$$
|f(z)| \geq\left(1-\left|b_{1}\right|\right) r-\left(\frac{1-\beta}{4}-\frac{1+\beta}{4}\left|b_{1}\right|\right) r^{2}, \quad|z|=r<1 .
$$

Proof. Let $f \in \overline{\mathscr{H}} \mathscr{C}_{s}(\beta)$. Taking the absolute value of $f$ we have

$$
\begin{aligned}
|f(z)| \leq & \left(1+\left|b_{1}\right|\right) r+\sum_{n=2}^{\infty}\left(\left|a_{n}\right|+\left|b_{n}\right|\right) r^{n} \\
\leq & \left(1+\left|b_{1}\right|\right) r+\sum_{n=2}^{\infty}\left(\left|a_{n}\right|+\left|b_{n}\right|\right) r^{2} \\
= & \left(1+\left|b_{1}\right|\right) r+\frac{1-\beta}{4} \sum_{n=2}^{\infty}\left(\frac{4}{1-\beta}\left|a_{n}\right|+\frac{4}{1-\beta}\left|b_{n}\right|\right) r^{2} \\
\leq & \left(1+\left|b_{1}\right|\right) r+ \\
& \frac{1-\beta}{4} \sum_{n=2}^{\infty}\left(\frac{n\left(2 n-\beta\left(1-(-1)^{n}\right)\right)}{2(1-\beta)}\left|a_{n}\right|+\frac{n\left(2 n+\beta\left(1-(-1)^{n}\right)\right)}{2(1-\beta)}\left|b_{n}\right|\right) r^{2} \\
\leq & \left(1+\left|b_{1}\right|\right) r+\frac{1-\beta}{4}\left(1-\frac{1+\beta}{1-\beta}\left|b_{1}\right|\right) r^{2} \\
= & \left(1+\left|b_{1}\right|\right) r+\left(\frac{1-\beta}{4}-\frac{1+\beta}{4}\left|b_{1}\right|\right) r^{2}
\end{aligned}
$$

and

$$
\begin{aligned}
|f(z)| \geq & \left(1-\left|b_{1}\right|\right) r-\sum_{n=2}^{\infty}\left(\left|a_{n}\right|+\left|b_{n}\right|\right) r^{n} \\
\geq & \left(1-\left|b_{1}\right|\right) r-\sum_{n=2}^{\infty}\left(\left|a_{n}\right|+\left|b_{n}\right|\right) r^{2} \\
= & \left(1-\left|b_{1}\right|\right) r-\frac{1-\beta}{4} \sum_{n=2}^{\infty}\left(\frac{4}{1-\beta}\left|a_{n}\right|+\frac{4}{1-\beta}\left|b_{n}\right|\right) r^{2} \\
\geq & \left(1-\left|b_{1}\right|\right) r- \\
& \frac{1-\beta}{4} \sum_{n=2}^{\infty}\left(\frac{n\left(2 n-\beta\left(1-(-1)^{n}\right)\right)}{2(1-\beta)}\left|a_{n}\right|+\frac{n\left(2 n+\beta\left(1-(-1)^{n}\right)\right)}{2(1-\beta)}\left|b_{n}\right|\right) r^{2} \\
\geq & \left(1-\left|b_{1}\right|\right) r-\frac{1-\beta}{4}\left(1-\frac{1+\beta}{1-\beta}\left|b_{1}\right|\right) r^{2} \\
= & \left(1-\left|b_{1}\right|\right) r-\left(\frac{1-\beta}{4}-\frac{1+\beta}{4}\left|b_{1}\right|\right) r^{2} .
\end{aligned}
$$

The bounds given in Theorem 2.3 for the functions $f=h+\bar{g}$ of the form (2) also hold for functions of the form (1) if the coefficient condition (5) is satisfied. The upper bound given 
for $f \in \overline{\mathscr{P}} \mathscr{C}_{s}(\beta)$ is sharp and the equality occurs for the function

$$
f(z)=z+\left|b_{1}\right| \bar{z}+\left(\frac{1-\beta}{4}-\frac{1+\beta}{4}\left|b_{1}\right|\right) \bar{z}^{2}, \quad\left|b_{1}\right| \leq \frac{1-\beta}{1+\beta} .
$$

Next, we determine the extreme points of closed hulls of $\overline{\mathscr{H}} \mathscr{C}_{s}(\beta)$ denoted by $\operatorname{clco} \overline{\mathscr{H}} \mathscr{C}_{s}(\beta)$.

Theorem 2.4. $f \in \operatorname{clco} \overline{\mathscr{H}} \mathscr{C}_{s}(\beta)$ if and only if $f(z)=\sum_{n=1}^{\infty}\left(X_{n} h_{n}+Y_{n} g_{n}\right)$ where

$$
\begin{aligned}
& h_{1}(z)=z, h_{n}(z)=z-\frac{2(1-\beta)}{n\left(2 n-\beta\left(1-(-1)^{n}\right)\right)} z^{n}(n=2,3, \ldots), \\
& g_{n}(z)=z-\frac{2(1-\beta)}{n\left(2 n+\beta\left(1-(-1)^{n}\right)\right)} \bar{z}^{n}(n=1,2, \ldots),
\end{aligned}
$$

$\sum_{n=1}^{\infty}\left(X_{n}+Y_{n}\right)=1, X_{n} \geq 0$ and $Y_{n} \geq 0$.

Proof. For $h_{n}$ and $g_{n}$ as given above, we may write

$$
\begin{aligned}
f(z) & =\sum_{n=1}^{\infty}\left(X_{n} h_{n}+Y_{n} g_{n}\right) \\
& =\sum_{n=1}^{\infty}\left(X_{n}+Y_{n}\right) z-\sum_{n=2}^{\infty} \frac{2(1-\beta)}{n\left(2 n-\beta\left(1-(-1)^{n}\right)\right)} X_{n} z^{n}-\sum_{n=1}^{\infty} \frac{2(1-\beta)}{n\left(2 n+\beta\left(1-(-1)^{n}\right)\right)} Y_{n} \bar{z}^{n} \\
& =z-\sum_{n=2}^{\infty} \frac{2(1-\beta)}{n\left(2 n-\beta\left(1-(-1)^{n}\right)\right)} X_{n} z^{n}-\sum_{n=1}^{\infty} \frac{2(1-\beta)}{n\left(2 n+\beta\left(1-(-1)^{n}\right)\right)} Y_{n} \bar{z}^{n} .
\end{aligned}
$$

Then

$$
\begin{aligned}
\sum_{n=2}^{\infty} & \frac{n\left(2 n-\beta\left(1-(-1)^{n}\right)\right)}{2(1-\beta)}\left|a_{n}\right|+\sum_{n=1}^{\infty} \frac{n\left(2 n+\beta\left(1-(-1)^{n}\right)\right)}{2(1-\beta)}\left|b_{n}\right| \\
= & \sum_{n=2}^{\infty} \frac{n\left(2 n-\beta\left(1-(-1)^{n}\right)\right)}{2(1-\beta)}\left(\frac{2(1-\beta)}{n\left(2 n-\beta\left(1-(-1)^{n}\right)\right)} X_{n}\right) \\
& +\sum_{n=1}^{\infty} \frac{n\left(2 n+\beta\left(1-(-1)^{n}\right)\right)}{2(1-\beta)}\left(\frac{2(1-\beta)}{n\left(2 n+\beta\left(1-(-1)^{n}\right)\right)} Y_{n}\right) \\
= & \sum_{n=2}^{\infty} X_{n}+\sum_{n=1}^{\infty} Y_{n} \\
= & 1-X_{1} \\
\leq & 1 .
\end{aligned}
$$

Therefore $f \in \operatorname{clco} \overline{\mathscr{H}} \mathscr{C}_{s}(\beta)$.

Conversely, suppose that $f \in \operatorname{clco} \overline{\mathscr{H}} \mathscr{C}_{s}(\beta)$. Set

$$
X_{n}=\frac{n\left(2 n-\beta\left(1-(-1)^{n}\right)\right)}{2(1-\beta)}\left|a_{n}\right|,(n=2,3,4, \ldots),
$$


and

$$
Y_{n}=\frac{n\left(2 n+\beta\left(1-(-1)^{n}\right)\right)}{2(1-\beta)}\left|b_{n}\right|,(n=1,2,3, \ldots),
$$

where $\sum_{n=1}^{\infty}\left(X_{n}+Y_{n}\right)=1$. Then

$$
\begin{aligned}
f(z) & =h(z)+\overline{g(z)} \\
& =z-\sum_{n=2}^{\infty}\left|a_{n}\right| z^{n}-\sum_{n=1}^{\infty}\left|b_{n}\right| \bar{z}^{n} \\
& =z-\sum_{n=2}^{\infty} \frac{2(1-\beta)}{n\left(2 n-\beta\left(1-(-1)^{n}\right)\right)} X_{n} z^{n}-\sum_{n=1}^{\infty} \frac{2(1-\beta)}{n\left(2 n+\beta\left(1-(-1)^{n}\right)\right)} Y_{n} \bar{z}^{n} \\
& =z+\sum_{n=2}^{\infty}\left(h_{n}(z)-z\right) X_{n}+\sum_{n=1}^{\infty}\left(g_{n}(z)-z\right) Y_{n} \\
& =\sum_{n=1}^{\infty}\left(X_{n} h_{n}+Y_{n} g_{n}\right) .
\end{aligned}
$$

For harmonic functions $f(z)=z-\sum_{n=2}^{\infty}\left|a_{n}\right| z^{n}-\sum_{n=1}^{\infty}\left|b_{n}\right| \bar{z}^{n}$ and $F(z)=z-\sum_{n=2}^{\infty}\left|A_{n}\right| z^{n}-$ $\sum_{n=1}^{\infty}\left|B_{n}\right| \bar{z}^{n}$, we define the convolution of $f$ and $F$ as

$$
(f \star F)(z)=z-\sum_{n=2}^{\infty}\left|a_{n} A_{n}\right| z^{n}-\sum_{n=1}^{\infty}\left|b_{n} B_{n}\right| \bar{z}^{n} .
$$

In the next theorem, we examine the convolution properties of the class $\overline{\mathscr{H}} \mathscr{C}_{s}(\beta)$.

Theorem 2.5. For $0 \leq \alpha \leq \beta<1$, let $f \in \overline{\mathscr{H}}_{\mathscr{C}}(\beta)$ and $F \in \overline{\mathscr{H}}_{\mathscr{C}_{s}}(\alpha)$. Then $(f \star F) \in$ $\overline{\mathscr{H}} \mathscr{C}_{s}(\beta) \subset \overline{\mathscr{H}} \mathscr{C}_{s}(\alpha)$.

Proof. Write $f(z)=z-\sum_{n=2}^{\infty}\left|a_{n}\right| z^{n}-\sum_{n=1}^{\infty}\left|b_{n}\right| \bar{z}^{n}$ and $F(z)=z-\sum_{n=2}^{\infty}\left|A_{n}\right| z^{n}-\sum_{n=1}^{\infty}\left|B_{n}\right| \bar{z}^{n}$. Then the convolution of $f$ and $F$ is given by (10).

Note that $\left|A_{n}\right| \leq 1$ and $\left|B_{n}\right| \leq 1$ since $F \in \overline{\mathscr{P}}_{\mathscr{C}}{ }_{s}(\alpha)$. Then we have

$$
\begin{aligned}
& \sum_{n=2}^{\infty} n\left[2 n-\beta\left(1-(-1)^{n}\right)\right]\left|a_{n}\right|\left|A_{n}\right|+\sum_{n=1}^{\infty} n\left[2 n+\beta\left(1-(-1)^{n}\right)\right]\left|b_{n}\right|\left|B_{n}\right| \\
& \leq \sum_{n=2}^{\infty} n\left[2 n-\beta\left(1-(-1)^{n}\right)\right]\left|a_{n}\right|+\sum_{n=1}^{\infty} n\left[2 n+\beta\left(1-(-1)^{n}\right)\right]\left|b_{n}\right| .
\end{aligned}
$$

Therefore, $(f \star F) \in \overline{\mathscr{H}} \mathscr{C}_{s}(\beta) \subset \overline{\mathscr{H}} \mathscr{C}_{s}(\alpha)$ since the right hand side of the above inequality is bounded by $2(1-\beta)$ while $2(1-\beta) \leq 2(1-\alpha)$.

Now, we determine the convex combination properties of the members of $\overline{\mathscr{H}_{C}}{ }_{s}(\beta)$.

Theorem 2.6. The class $\left.\overline{\mathscr{H}} \mathscr{C}_{s} \beta\right)$ is closed under convex combination.

Proof. For $i=1,2,3, \ldots$, suppose that $f_{i} \in \overline{\mathscr{C}} \mathscr{C}_{s}(\beta)$ where $f_{i}$ is given by

$$
f_{i}(z)=z-\sum_{n=2}^{\infty}\left|a_{n, i}\right| z^{n}-\sum_{n=1}^{\infty}\left|b_{n, i}\right| \bar{z}^{n} .
$$


For $\sum_{i=1}^{\infty} c_{i}=1,0 \leq c_{i} \leq 1$, the convex combinations of $f_{i}$ may be written as

$$
\begin{aligned}
\sum_{i=1}^{\infty} c_{i} f_{i}(z) & =c_{1} z-\sum_{n=2}^{\infty} c_{1}\left|a_{n, 1}\right| z^{n}-\sum_{n=1}^{\infty} c_{1}\left|b_{n, 1}\right| \bar{z}^{n}+c_{2} z-\sum_{n=2}^{\infty} c_{2}\left|a_{n, 2}\right| z^{n}-\sum_{n=1}^{\infty} c_{2}\left|b_{n, 2}\right| \bar{z}^{n} \ldots \\
& =z \sum_{i=1}^{\infty} c_{i}-\sum_{n=2}^{\infty}\left(\sum_{i=1}^{\infty} c_{i}\left|a_{n, i}\right|\right) z^{n}-\sum_{n=1}^{\infty}\left(\sum_{i=1}^{\infty} c_{i}\left|b_{n, i}\right|\right) \bar{z}^{n} \\
& =z-\sum_{n=2}^{\infty}\left(\sum_{i=1}^{\infty} c_{i}\left|a_{n, i}\right|\right) z^{n}-\sum_{n=1}^{\infty}\left(\sum_{i=1}^{\infty} c_{i}\left|b_{n, i}\right|\right) \bar{z}^{n} .
\end{aligned}
$$

Next, consider

$$
\begin{aligned}
\sum_{n=2}^{\infty}\left(n\left[2 n-\beta\left(1-(-1)^{n}\right)\right]\left|\sum_{i=1}^{\infty} c_{i}\right| a_{n, i}||\right)+\sum_{n=1}^{\infty}\left(n\left[2 n+\beta\left(1-(-1)^{n}\right)\right]\left|\sum_{i=1}^{\infty} c_{i}\right| b_{n, i}||\right) \\
=c_{1} \sum_{n=2}^{\infty} n\left[2 n-\beta\left(1-(-1)^{n}\right)\right]\left|a_{n, 1}\right|+\cdots+c_{m} \sum_{n=2}^{\infty} n\left[2 n-\beta\left(1-(-1)^{n}\right)\right]\left|a_{n, m}\right|+\cdots \\
\quad+c_{1} \sum_{n=1}^{\infty} n\left[2 n+\beta\left(1-(-1)^{n}\right)\right]\left|b_{n, 1}\right|+\cdots+c_{m} \sum_{n=1}^{\infty} n\left[2 n+\beta\left(1-(-1)^{n}\right)\right]\left|b_{n, m}\right|+\cdots \\
=\sum_{i=1}^{\infty} c_{i}\left\{\sum_{n=2}^{\infty} n\left[2 n-\beta\left(1-(-1)^{n}\right)\right]\left|a_{n, i}\right|+\sum_{n=1}^{\infty} n\left[2 n+\beta\left(1-(-1)^{n}\right)\right]\left|b_{n, i}\right|\right\} .
\end{aligned}
$$

Now, $f_{i} \in \overline{\mathscr{H}} \mathscr{C}_{s}(\beta)$, therefore from Theorem 2.2, we have

$$
\sum_{n=2}^{\infty} n\left[2 n-\beta\left(1-(-1)^{n}\right)\right]\left|a_{n, i}\right|+\sum_{n=1}^{\infty} n\left[2 n+\beta\left(1-(-1)^{n}\right)\right]\left|b_{n, i}\right| \leq 2(1-\beta) .
$$

Hence

$$
\begin{aligned}
& \sum_{n=2}^{\infty}\left(n\left[2 n-\beta\left(1-(-1)^{n}\right)\right]\left|\sum_{i=1}^{\infty} c_{i}\right| a_{n, i} \mid\right)+\sum_{n=1}^{\infty}\left(n\left[2 n+\beta\left(1-(-1)^{n}\right)\right]\left|\sum_{i=1}^{\infty} c_{i}\right| b_{n, i} \mid\right) \\
& \leq 2(1-\beta) \sum_{i=1}^{\infty} c_{i} \\
& =2(1-\beta) .
\end{aligned}
$$

By using Theorem 2.2 again, we have $\sum_{i=1}^{\infty} c_{i} f_{i} \in \overline{\mathscr{H}} \mathscr{C}_{s}(\beta)$.

\section{References}

[1] Ahuja, O. P. and Jahangiri, J. M. (2001). A subclass of harmonic univalent functions, J. of Natural Geometry 20. 45-56

[2] Clunie, J. and Sheil Small, T. (1984). Harmonic univalent functions, Ann. Acad. Aci. Fenn. Ser. A. I. Math. 9. 3-25

[3] Darus, M., Halim, S. A. and Janteng, A. (2006). Properties of harmonic functions which are starlike of order $\beta$ with respect to symmetric points, Proceeding of The First International Conference on Mathematics and Statistics, 19-24 


\section{REFERENCES}

[4] Das, R. N. and Singh, P. (1977). On subclasses of schlicht mapping, Indian J. Pure Appl. Math. 8. 864-872

[5] Jahangiri, J. M. (1998). Coefficient bounds and univalence criteria for harmonic functions with negative coefficients, Ann. Univ. Marie Curie. Sklodowska. Sec. A 52. 57-66

[6] Jahangiri, J. M. (1999). Harmonic functions starlike in the unit disk, J. Math. Anal. Appl. 235. 470477

[7] Kim, Y. C., Jahangiri, J. M. and Choi, J. H. (2002). Certain convex harmonic functions, Int. J. Math. Math. Sci. 29, 459-465

[8] Sakaguchi, K. (1959). On a certain univalent mapping, J. Math. Soc. Japan, 11 72-75

[9] Silverman, H. (1998). Harmonic univalent functions with negative coefficients. J. Math. Anal. Appl. 220, 283-289

[10] Silverman, H. and Silvia, E. M. (1999). Subclasses of harmonic univalent functions, New Zealand J. Math. 28, 275-284

School of Science and Technology, Universiti Malaysia Sabah, Locked Bag No.2073, 88999 Kota Kinabalu, Sabah, Malaysia.

E-mail:aini_jg@ums.edu.my

Institute of Mathematical Sciences, Universiti Malaya, 50603 Kuala Lumpur, Malaysia.

E-mail: suzeini@um.edu.my 